\title{
Systemic Infection by Fusarium verticillioides in Maize Plants Grown Under Three Temperature Regimes
}

\author{
A. Murillo-Williams, Centro para Investigaciones en Granos y Semillas, Universidad de Costa Rica, San José, \\ Costa Rica; and G. P. Munkvold, Seed Science Center and Department of Plant Pathology, Iowa State University, \\ Ames, IA 50011
}

\begin{abstract}
Murillo-Williams, A., and Munkvold, G. P. 2008. Systemic infection by Fusarium verticillioides in maize plants grown under three temperature regimes. Plant Dis. 92:1695-1700.

Fusarium verticillioides causes seedling decay, stalk rot, ear rot, and mycotoxin contamination (primarily fumonisins) in maize. Systemic infection of maize plants by F. verticillioides can lead to kernel infection, but the frequency of this phenomenon has varied widely among experiments. Variation in the incidence of systemic infection has been attributed to environmental factors. In order to better understand the influence of environment, we investigated the effect of temperature on systemic development of $F$. verticillioides during vegetative and reproductive stages of plant development. Maize seeds were inoculated with a green fluorescent protein-expressing strain of $F$. verticillioides, and grown in growth chambers under three different temperature regimes. In the vegetative-stage and reproductive-stage experiments, plants were evaluated at tasseling (VT stage), and at physiological maturity (R6 stage), respectively. Independently of the temperature treatment, F. verticillioides was reisolated from nearly $100 \%$ of belowground plant tissues. Frequency of reisolation of the inoculated strain declined acropetally in aboveground internodes at all temperature regimes. At VT, the high-temperature treatment had the highest systemic development of $F$. verticillioides in aboveground tissues. At R6, incidence of systemic infection was greater at both the high- and low-temperature regimes than at the averagetemperature regime. F. verticillioides was isolated from higher internodes in plants at R6, compared to stage VT. The seed-inoculated strain was recovered from kernels of mature plants, although incidence of kernel infection did not differ significantly among treatments. During the vegetative growth stages, temperature had a significant effect on systemic development of $F$. verticillioides in stalks. At R6, the fungus reached higher internodes in the high-temperature treatment, but temperature did not have an effect on the incidence of kernels (either symptomatic or asymptomatic) or ear peduncles infected with the inoculated strain. These results support the role of high temperatures in promoting systemic infection of maize by $F$. verticillioides, but plant-to-seed transmission may be limited by other environmental factors that interact with temperature during the reproductive stages.
\end{abstract}

Additional keywords: endophytic infection, Fusarium moniliforme

Fusarium verticillioides (Sacc.) Nirenberg (syn. F. moniliforme J. Sheld.) is the most prevalent fungus associated with maize (Zea mays L.) $(18,23,29)$, causing seed rot, seedling diseases, ear rot, stalk rot, and reductions in crop yield worldwide $(17,19,22,35,41)$. $F$. verticillioides produces a wide range of mycotoxins that includes fusaric acid, fusarins, and fumonisins (6). Fumonisins are the most prevalent (25) of these mycotoxins, and they have been associated with esophageal cancer in humans, pulmonary edema in pigs, leukoencephalomalacia in horses, and cancer-promoting activity in rats $(12,18,39)$.

Corresponding author: G. P. Munkvold

E-mail: munkvold@iastate.edu

Accepted for publication 16 September 2008.

doi:10.1094/PDIS-92-12-1695

(C) 2008 The American Phytopathological Society
Major pathways for kernel infection and ear rot caused by $F$. verticillioides are through silks and insect wounds $(27,30,38)$. The fungus also colonizes maize stalks systemically without causing visible disease symptoms $(11,13,26,27)$, and $F$. verticillioides strains can be vertically transmitted through seed-to-plant transmission and systemic stalk infection, followed by plantto-seed transmission (27).

Previous studies of systemic infection by $F$. verticillioides from inoculated seed have produced a variety of results. Kedera et al. (13) conducted the first study that involved vegetative compatibility as a marker to differentiate the seed-inoculated strain of $F$. verticillioides from other strains in field experiments. At plant maturity, the seed-inoculated strain was recovered from the crown of $65 \%$ of plants, and recovery from aboveground plant tissues decreased acropetally. These authors reported $8.4 \%$ of kernels yielded the seedinoculated strain. Munkvold and Carlton (26) and Munkvold et al. (27) confirmed that seed infection can lead to kernel colonization through systemic development of F. verticillioides, although the recovery of seed-inoculated strains from ears varied greatly. Munkvold et al. (27) described the transmission of $F$. verticillioides from seed-to-kernels in four steps that include (i) seed to seedling transmission, (ii) colonization of the stalk, (iii) movement into the ear, and (iv) spread within the ear. These experiments showed that seedseedling transmission occurs frequently, but limited movement from the crown to higher internodes in the stalk seemed to limit systemic colonization of the kernels.

Temperature may be one factor that determines the extent of invasion of the stalk by ear rot fungi (19). F. verticillioides is more common in regions with hot and dry growing conditions $(1,7,9,34,38)$, especially before or during pollination $(4,31)$. $F$. verticillioides grows well at temperatures above $26^{\circ} \mathrm{C}$ (32). The calculated optimal and maximum temperatures for growth of $F$. verticillioides are 31 and $35^{\circ} \mathrm{C}$, respectively, and 22 to $24^{\circ} \mathrm{C}$ is the suggested minimum range for growth (36). There are reports of $F$. verticillioides growing at temperatures below $20^{\circ} \mathrm{C}$, although the effect of temperature in these experiments was influenced by water availability in the substrate $(10,21)$. This wide range of temperatures extends beyond those optimal for maize growth, so that $F$. verticillioides can function at temperatures under which maize plants may experience stress. We hypothesize that temperature may be the limiting factor for stalk-to-kernel movement of $F$. verticillioides by affecting both plant and fungal growth. The objective of this work was to assess the effect of temperature on the systemic movement of $F$. verticillioides in maize plants.

\section{MATERIALS AND METHODS}

Two sets of experiments (vegetative stage and reproductive stage) were conducted in order to assess the effects of temperature on systemic development of $F$. verticillioides during maize vegetative and reproductive stages.

F. verticillioides was transformed with a gene for green fluorescent protein (GFP) expression and hygromycin $\mathrm{B}$ resistance (transformant TXI-79) (40). When compared to the wild type, $F$. verticillioides TXI-79 growth and the ability to cause stalk infection were not affected by the 
transformation (40). Inoculum from TXI79 was prepared from 7-day-old cultures grown in complete medium including $\mathrm{xy}-$ lose (16). The inoculum consisted of conidia obtained by flooding a petri dish with $4 \mathrm{ml}$ of sterile distilled water and then pouring it through sterile cheesecloth into a sterile Erlenmeyer flask. The concentration of the inoculum suspension was $10^{6}$ conidia $\mathrm{ml}^{-1}(40)$.

Fungal colonies were confirmed as TXI79 when GFP expression was observed in mycelium growing from seeds and plant tissues. Fungal colonies were examined with a Zeiss Axioplan 2 compound microscope (Carl Zeiss Microimaging, Thornwood, NY) fitted with epifluorescence $\mathrm{Hg}$ 100w lamp, and a filter cube for GFP (excitation wavelength $=395 \mathrm{~nm}$; emission wavelength $=598 \mathrm{~nm}$ ).

Vegetative-stage experiments. The maize hybrid 'Cargill 1077' (Cargill, Minneapolis, MN; currently Mycogen Seeds, Indianapolis, IN) was selected because of short stature and early maturity. Seeds were surface disinfested with a solution of $0.5 \%$ sodium hypochlorite for $2 \mathrm{~min}$ and then washed with sterile distilled water for
30 s. The dry seeds were placed in Erlenmeyer flasks with $70 \mathrm{ml}$ of $F$. verticillioides TXI-79 inoculum or sterile distilled water (controls), and then placed on an orbital shaker at $120 \mathrm{r} \cdot \mathrm{s}^{-1}$ for $12 \mathrm{~h}$. Before planting, inoculated and control seeds were placed in separate hoods to dry for $2 \mathrm{~h}$ (40).

To test incidence of seed infection, 25 inoculated and 25 noninoculated seeds from each batch were surface disinfested with a $0.5 \%$ sodium hypochlorite solution for $5 \mathrm{~min}, 70 \%$ ethanol for $3 \mathrm{~min}$, and sterile distilled water for $2 \mathrm{~min}$, and then placed on Nash-Snyder medium (NSM) (28) with $2 \mathrm{mg} \cdot$ liter $^{-1}$ hygromycin B (five seeds per petri dish). Another 25 inoculated seeds were placed on the same medium without surface disinfestation. The seeds were incubated in the dark at $25^{\circ} \mathrm{C}$ for 5 days to allow fungal growth. The proportion of seeds with TXI-79 colonies was recorded.

Clay pots (15.3-cm-diameter) containing 2 parts soil: 1 part vermiculite: 1 part sphagnum peat (by volume) were planted with either inoculated seeds or noninoculated seeds (two seeds per pot), randomly

Table 1. Programmed and recorded mean temperatures in growth chambers during first weeks of vegetative (VT) and reproductive (R6) stage experiments, before temperature treatments (low, average, and high) were imposed ${ }^{\mathrm{a}}$

\begin{tabular}{|c|c|c|c|c|c|c|}
\hline \multirow[b]{2}{*}{ Week } & \multicolumn{2}{|c|}{$\begin{array}{c}\text { Programmed temperatures } \\
\left({ }^{\circ} \mathbf{C}\right)\end{array}$} & \multicolumn{2}{|c|}{$\begin{array}{l}\text { Recorded temperatures } \\
\qquad\left({ }^{\circ} \mathbf{C}\right)\end{array}$} & \multicolumn{2}{|c|}{ Standard error } \\
\hline & Day & Night & Day & Night & Day & Night \\
\hline 1 & 23 & 17 & 23.3 & 17.4 & 0.1 & 0.4 \\
\hline 2 & 24 & 17.5 & 24.1 & 17.2 & 0.1 & 0.1 \\
\hline 3 & 27 & 18 & 26.1 & 18.1 & 0.5 & 0.4 \\
\hline 4 (V6) & 28 & 19 & 27.9 & 19.8 & 0.6 & 0.7 \\
\hline 5 & 30 & 21 & 29.1 & 21.3 & 0.5 & 0.1 \\
\hline 6 & 31 & 24 & 29 & 21.7 & 0.6 & 0.5 \\
\hline 7 & 32 & 25 & 30.2 & 23.3 & 0.4 & 0.4 \\
\hline 8 & 33 & 25 & 30.5 & 23.9 & 0.4 & 0.4 \\
\hline 9 (VT) & 34 & 26 & 30.0 & 23.9 & 0.4 & 0.7 \\
\hline
\end{tabular}

a Maize plants in VT experiment were exposed to these temperatures for 4 weeks, until they reached V6, and plants in R6 experiment grew under this temperature regime for 9 weeks, until they reached VT stage. arranged in each of three growth chambers. There were 25 pots planted with inoculated seeds and 10 pots with noninoculated seeds in each growth chamber. After emergence, pots were thinned to one plant per pot, so that 25 plants from inoculated seeds and 10 plants from noninoculated seeds were evaluated from each growth chamber.

Treatments consisted of three temperature regimes that simulated a range of conditions in Iowa during the growing season (39), based on a 10-year study of temperatures in the state (7). Each growth chamber represented a different treatment, and temperatures were changed weekly to follow the seasonal progression (Tables 1 and 2). Treatments consisted of: (i) average (based on seasonal average temperatures in Iowa); (ii) low (two standard deviations below average); and (iii) high (two standard deviations above average). Day temperatures were maintained during a 14-h light period and night temperatures during a 10-h dark period. The light source was a combination of fluorescent light and incandescent light bulbs. The recorded mean photosynthetically active radiation (PAR) intensity was $350 \mu \mathrm{mol} \cdot \mathrm{m}^{-2} \cdot \mathrm{s}^{-1}$. Data $\log$ gers were placed in the growth chambers to record air and soil temperatures.

Plants in all three treatments grew until growth stage V6 (33) (4 weeks after planting) under the high-temperature regime (Table 1). Five weeks after planting, the three temperature treatments were imposed, one in each growth chamber, until sampling. Treatments were randomized among the growth chambers for replications of the experiments, and each temperature treatment was replicated three times, so that a total of 75 inoculated and 30 noninoculated plants were evaluated for each temperature treatment. The recorded and programmed air temperatures in the growth chambers are shown in Table 2.

When plants reached the tasseling stage (VT) (8 to 9 weeks after planting), their height from the soil line to the last fully

Table 2. Programmed and recorded temperatures in growth chambers for each temperature treatment (low, average, and high) of the vegetative stage (VT) and reproductive stage (R6) experiments ${ }^{\mathrm{a}}$

\begin{tabular}{|c|c|c|c|c|c|c|c|c|c|c|c|c|}
\hline \multirow[b]{3}{*}{ Week } & \multicolumn{6}{|c|}{ Programmed temperatures $\left({ }^{\circ} \mathbf{C}\right)$} & \multicolumn{6}{|c|}{ Recorded temperatures $\left({ }^{\circ} \mathbf{C}\right)$} \\
\hline & \multicolumn{2}{|c|}{ Low } & \multicolumn{2}{|c|}{ Average } & \multicolumn{2}{|c|}{ High } & \multicolumn{2}{|c|}{ Low } & \multicolumn{2}{|c|}{ Average } & \multicolumn{2}{|c|}{ High } \\
\hline & Day & Night & Day & Night & Day & Night & Day & Night & Day & Night & Day & Night \\
\hline 5 & 15.0 & 11.0 & 24.0 & 18.0 & 31.0 & 24.0 & 15.9 & 10.0 & 23.4 & 17.9 & 29.7 & 23.5 \\
\hline 6 & 17.0 & 13.0 & 25.5 & 19.0 & 32.0 & 25.0 & 17.4 & 11.5 & 24.3 & 19.2 & 30.9 & 24.8 \\
\hline 7 & 18.0 & 14.0 & 27.0 & 20.5 & 33.0 & 25.5 & 18.7 & 13.5 & 25.7 & 20.1 & 31.4 & 25.6 \\
\hline 8 & 20.0 & 16.0 & 28.0 & 21.0 & 34.0 & 26.0 & 20.8 & 15.0 & 28.5 & 18.0 & 31.9 & 26.9 \\
\hline $9(\mathrm{VT})$ & 22.0 & 17.0 & & & & & 22.0 & 16.5 & & & & \\
\hline 10 & 17.0 & 13.0 & 24.0 & 18.0 & 31.0 & 24.0 & 17.0 & 13.6 & 24.5 & 19.5 & 30.7 & 25.5 \\
\hline 11 & 18.0 & 14.0 & 25.5 & 19.0 & 35.0 & 27.0 & 17.5 & 14.0 & 25.5 & 20.6 & 31.9 & 26.0 \\
\hline 12 & 20.0 & 16.0 & 27.0 & 20.5 & 35.5 & 27.5 & 19.1 & 16.6 & 27.7 & 20.6 & 32.3 & 27.1 \\
\hline 13 & 22.0 & 17.0 & 28.0 & 21.0 & 36.0 & 28.0 & 21.3 & 17.5 & 28.6 & 21.6 & 33.2 & 25.2 \\
\hline 14 & 23.0 & 17.5 & 28.5 & 21.5 & 34.0 & 26.0 & 22.3 & 18.6 & 29.3 & 23.3 & 33.3 & 27.4 \\
\hline 15 & 23.5 & 18.0 & & & & & 23.9 & 19.0 & & & & \\
\hline $16(\mathrm{R} 6)$ & 24.0 & 19.0 & & & & & 24.0 & 19.6 & & & & \\
\hline
\end{tabular}

${ }^{\mathrm{a}}$ In the VT experiment, treatments were imposed 5 weeks after planting, the period of time between growth stage V6 (4 weeks after planting) and growth stage VT. In the reproductive stage experiment, treatments were imposed 9 weeks after planting, the period of time between growth stage VT and growth stage R6. 
developed leaf was recorded, and they were removed from the pots. The stems were then cut into $20-\mathrm{cm}$ segments and surface disinfested with a $0.5 \%$ sodium hypochlorite solution for $5 \mathrm{~min}, 70 \%$ ethanol for $3 \mathrm{~min}$, and sterile distilled water for $2 \mathrm{~min}$. A $2-\mathrm{cm}$ portion from the center of each internode of each plant was split longitudinally, and both segments were placed in culture plates with the cut part of the stalk in contact with the medium (NashSnyder with hygromycin). The shank (peduncle of the ovulate inflorescence), crown, mesocotyls, and five 2-cm-long pieces of the primary root were also placed in culture. The material was kept in the dark for 6 days at room temperature, and microscopically examined for GFPexpressing fungal colonies. Plant segments from which $F$. verticillioides TXI-79 strain grew were scored as positive, and the percentage of positives was calculated for each plant tissue.

Reproductive-stage experiments. Plants grown from inoculated and noninoculated seeds in all three temperature treatments grew until growth stage VT (9 weeks after planting) under a high-temperature regime (Table 1). Ten weeks after planting, the three temperature treatments (Table 2) were imposed, one in each growth chamber. Tassels were removed from plants grown from inoculated seeds. Pollen from noninoculated plants was collected, and all plants were hand-pollinated at least two times, 2 to 3 days after silk emergence.

When plants reached growth stage R6 (physiological maturity), their height from the soil line to the flag leaf was recorded. The plants were then removed from the pots, aseptically cut into $20-\mathrm{cm}$ segments, and surface disinfested with a $0.5 \%$ sodium hypochlorite solution for $5 \mathrm{~min}, 70 \%$ ethanol for $3 \mathrm{~min}$, and sterile distilled water for $2 \mathrm{~min}$. The same plant tissues as in the vegetative stage experiments were placed in culture. From the harvested ears, 25 symptomless kernels were arbitrarily selected and placed in culture, as well as all kernels showing symptoms or signs of Fusarium ear rot (starburst or white mycelium). If there were fewer than 25 kernels on an ear, all the kernels were placed in culture. The material was kept in the dark for 6 days at room temperature $\left(25^{\circ} \mathrm{C}\right)$ and then examined microscopically for GFP expression. Plant segments and kernels from which $F$. verticillioides TXI-79 strain grew were scored as positive, and the total of positives was recorded for each plant tissue type.

We measured the extent of $F$. verticillioides development in stalks in two ways: by recording the total number of colonized aboveground internodes for each replication of each treatment combination, and by recording the highest internode from which the fungus was recovered in each plant. The means of the plant-by-plant values were calculated for each replication of temperature by inoculation combination. There were three replications for each temperature; however, data from the second replication of the average temperature treatment of the reproductive stage experiment were not utilized due to technical problems with the growth chamber.

Effects of temperature on plant height, percentage of internodes colonized, maximum internode reached by the fungus, infected ear peduncles, and proportion of infected kernels were each fitted to a mixed linear model having fixed effects for temperature, treatment, and their interaction, and a random effect for replicate nested within temperature (used for testing the main effects of temperature). Least significant difference (LSD) tests at the 0.05 level were calculated to compare means. Analyses were conducted with the
MIXED procedure of SAS ver. 9.1 (SAS, Cary, NC).

\section{RESULTS}

The test for incidence of seed infection indicated that $100 \%$ of inoculated seeds placed in culture were positive for $F$. verticillioides TXI-79 with and without surface disinfestation. Inoculation treatment did not affect time to seedling emergence.

The period of time for plants to reach the VT stage was 8 weeks for the hightemperature treatments and 9 weeks for the average- and low-temperature treatments (Table 2). In the reproductive-stage experiment, the time for the plants to reach physiological maturity was 14 weeks for the high- and average-temperature treatments; and 17 weeks for plants under the low-temperature treatment (Table 2).

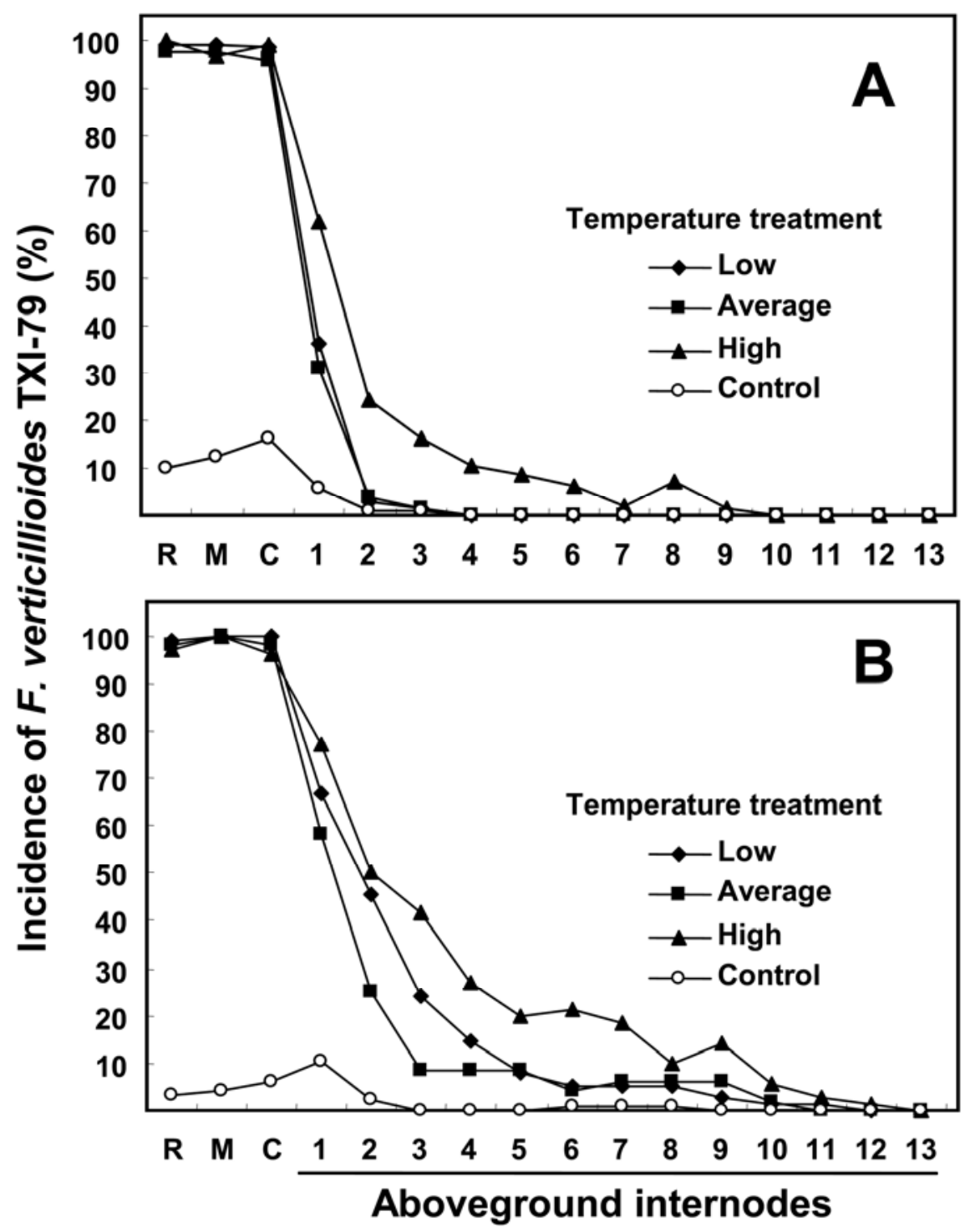

Fig. 1. Incidence of Fusarium verticillioides strain TXI-79 isolated from roots, mesocotyls, crowns, and internodes of maize plants grown from inoculated seeds in growth chambers. A, Temperature treatments (low, average, or high) were imposed from growth stages V6 (4 weeks after planting) to tasseling (VT). B, Temperature treatments were imposed from growth stage VT to physiological maturity (R6). Mean from inoculated seeds at average temperatures is based on two replications; all others are based on three. $\mathrm{R}=$ root, $\mathrm{M}=$ mesocotyl, $\mathrm{C}=$ crown. Control line represents mean incidence of F. verticillioides TXI-79 in noninoculated plants for all three treatments. 
Plant height (data not shown) was not affected by inoculation or temperature treatment at growth stages VT or R6. In plant vegetative tissues, differences in incidence of $F$. verticillioides TXI-79 strain between inoculated plants and noninoculated plants were always significant, for all temperature treatments at VT and R6 growth stages $(P<0.05)$. The trend in frequency of isolation from all vegetative plant tissues was the same for plants sampled at VT (Fig. 1A) and R6 (Fig. 1B). The highest frequency of isolation (95 to $100 \%$ ) of $F$. verticillioides TXI-79 strain

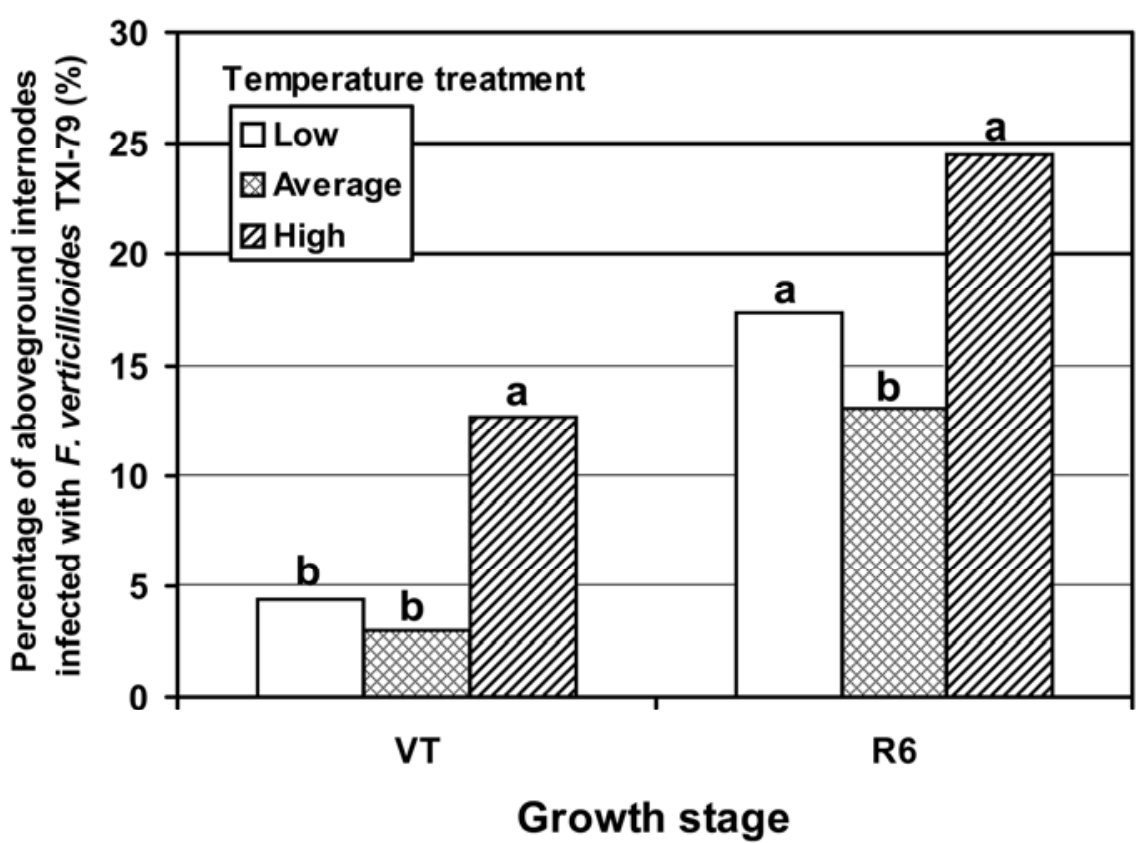

Fig. 2. Incidence of Fusarium verticillioides strain TXI-79 in aboveground internodes of maize plants grown from inoculated seeds in growth chambers. Temperature treatments were imposed from growth stage V6 (4 weeks after planting) to tasseling (VT), and from VT to physiological maturity (R6). Mean from inoculated seeds at average temperatures is based on two replications; all others are based on three. Bars with the same letter are not significantly different from each other at the 0.05 probability level.
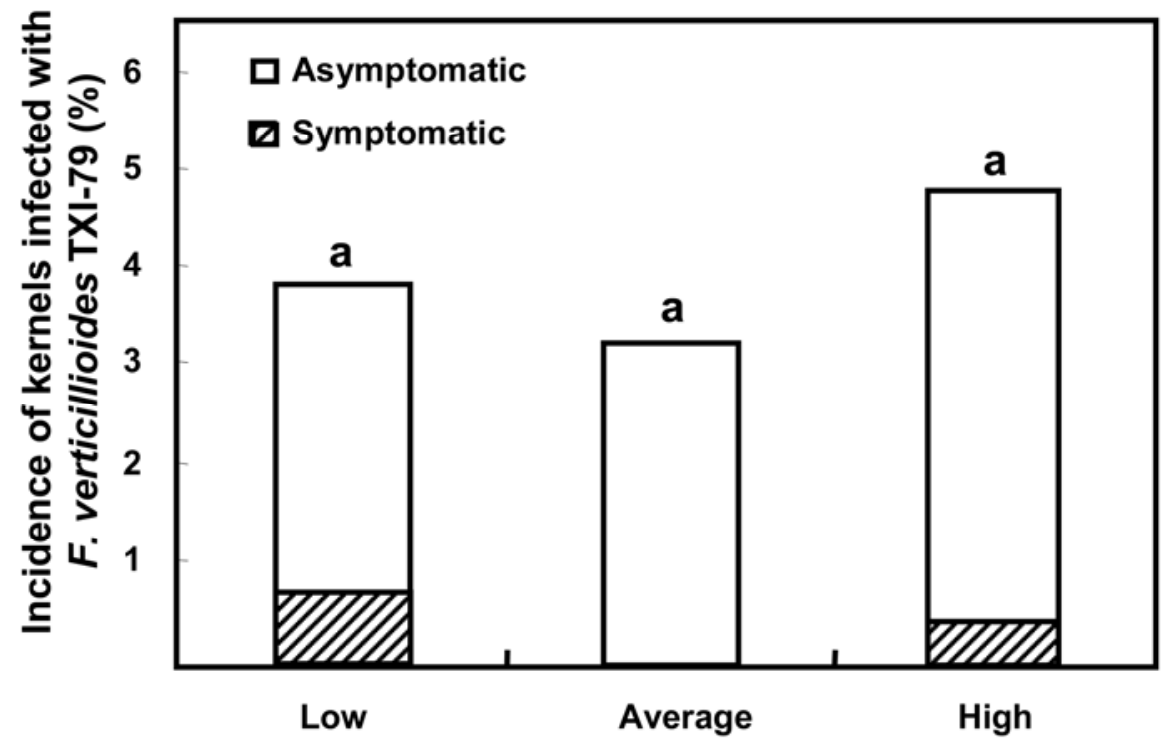

Temperature treatment

Fig. 3. Incidence of Fusarium verticillioides strain TXI-79 from kernels of maize plants grown from inoculated seeds and sampled at physiological maturity (R6) in growth chambers. Temperature treatments (low, average, or high) were imposed from growth stage tasseling (VT) to R6. Shaded area represents the percentage of kernels showing signs (white mycelium) and symptoms (starburst and rotting). Mean for the average temperature treatment is based on two replications; all others are based on three. Bars with the same letter are not significantly different from each other at the 0.05 probability level.

occurred from the belowground tissues (crown, mesocotyls, and primary root) for all temperature treatments and growth stages, with a steep decline in frequency of recovery of the fungus in progressively higher aboveground internodes. In the VT experiment, the percentage of aboveground colonized internodes was greater under the high-temperature treatment; however, in the R6 experiment, both the high- and lowtemperature treatments had greater percentages of colonized internodes than the average-temperature treatment (Fig. 2).

The fungus reached higher internodes in the high-temperature treatment (second aboveground internode) than for the average- and low-temperature treatments, where the fungus remained in the crown and first aboveground internode. In the R6 experiments, $F$. verticillioides TXI-79 also reached higher internodes under the hightemperature treatment (fourth aboveground internode), while infection remained in the second aboveground internodes in the lowand average-temperature treatments.

Harvested ears in the R6 experiment were mostly associated with the seventh internode. The percentage of plants with the seventh internode infected was 5,6 , and $19 \%$ for the low-, average-, and hightemperature treatments, respectively. Under the high-temperature treatment, the percentage was significantly higher than under the low- and average-temperature treatments $(P=0.01)$.

None of the peduncles from immature ears in the vegetative stage experiment was infected with $F$. verticillioides TXI-79. At maturity, infection by $F$. verticillioides TXI-79 was $37 \%$ of the ear-peduncles of inoculated plants for the high-temperature treatment, $22 \%$ for the average-, and $21 \%$ for the low-temperature treatment, but these means were not significantly different. Contamination of peduncles with $F$. verticillioides TXI-79 was detected in the control plants (7\%); however, this percentage was significantly different from that of the inoculated plants $(P \leq 0.0001)$.

The percentage of kernels infected with F. verticillioides TXI-79 ranged between 3 and $5 \%$ but did not differ among temperature treatments (Fig. 3). From the kernels that tested positive for $F$. verticillioides TXI-79, 13 and 6\% showed signs or symptoms for the low- and high-temperature treatments, respectively (less than $1 \%$ of the total number of kernels), and the remaining kernels had symptomless infections.

\section{DISCUSSION}

There were similarities in our results with other studies on systemic infection in plants from inoculated seeds $(5,13,26$, 27,40 ). Wilke et al. (40) showed that seedto-seedling transmission of $F$. verticillioides and colonization of plants up to stage V6 occurred equally over a wide range of temperatures. Seedborne inocu- 
lum infected the seedlings and remained primarily in the belowground tissues. In our studies, seed-to-seedling transmission also occurred at all temperature treatments in both growth stages. The fungus moved from the belowground tissues to aboveground internodes as high as the 12th internode aboveground (Fig. 1), and the frequency of isolation from internodes decreased with height above the soil. The fungus reached higher internodes in the reproductive stage experiment than in the vegetative stage experiment. These results are consistent with those of Kedera et al. (13), Cotten (5), and Munkvold and Carlton (26) in plants at physiological maturity. Growth stage seems to be closely related to the extent of movement of $F$. verticillioides within the plant; the fungus moves into and through aboveground tissues primarily after pollination (15). Temperature influenced the extent of colonization and the total number of internodes infected with the seed-inoculated strain (Fig. 2), with higher temperatures favoring the most extensive colonization. This effect was most evident for high temperatures applied during the late vegetative stages; during reproductive stages, the trend was not as clearly defined, and it may be necessary to conduct experiments with greater statistical power in order to clarify reproductivestage temperature effects.

We isolated $F$. verticillioides TXI-79 from $22 \%$ of the ears, $27 \%$ of peduncles, and approximately $5 \%$ of the kernels for all inoculated plants at all temperature treatments. These results are similar to those obtained by Kedera et al. (13), Munkvold et al. (27), and Cotten (5), who isolated seed-inoculated strains from 8, 10, and $16 \%$ of the kernels, but they differ from results obtained by Munkvold and Carlton (26), who isolated a seedinoculated strain from up to $78 \%$ of ears and $29 \%$ of kernels in one experiment. Plants in our experiments did not show stalk rot symptoms at the time of sampling, and just a low percentage of the kernels were symptomatic (Fig. 3). When discoloration was present in the stalk tissues, it was often free of the inoculated strain or other fungi, as described by Kingsland and Wernham (14).

As suggested by several researchers, factors other than temperature, such as rainfall or water availability, or the interaction of several factors, may determine the extent of colonization of maize by $F$. verticillioides $(7,20,23,24,34)$. The ability of $F$. verticillioides to grow under a wide range of temperatures $(20,32)$ is one factor that may have contributed to the lack of temperature effect on reisolation of the seedinoculated strain from peduncles and kernels of plants at physiological maturity. When ears were sampled at R6, the plants had been exposed to temperatures that ranged from $13.6^{\circ} \mathrm{C}$ (dark period, lowtemperature treatment) to $33.3^{\circ} \mathrm{C}$ (light period, high-temperature treatment). This range includes the optimal temperatures for growth of maize and $F$. verticillioides; and the range does not exceed the maximum temperatures for growth and development of either organism. Temperatures higher than $35^{\circ} \mathrm{C}$ may have been needed in order to induce a plant stress needed for enhanced colonization of kernels, although temperatures in that range have limited relevance to Iowa growing conditions, and approach the temperatures for maximum growth and development of $F$. verticillioides. Also, the effect of high temperature on $F$. verticillioides development in maize may be dependent on an interaction with plant water deficits $(5,20,23,24,34)$.

Corn growth and development is closely related to accumulated modified growing degree days (MGDD), and delays in plant development and reduced yield have been associated with cool temperatures early in the season. In this experiment, the number of days required to reach VT varied according to temperature, but MGDD required to reach VT were similar among treatments and were in the range reported in the literature (3). However, all plants reached V6 stage at the same time, since growing conditions were the same before temperature treatments were imposed (370 MGDD). The lack of a temperature effect on height could be associated with earlier vegetative stages being more sensitive to very low or very high temperatures (3) than later vegetative stages.

The seed-inoculated strain was also isolated from a small percentage of the control plants. Possible sources of contamination of the noninoculated plants are splashing water, soil particles, and infected senescent leaves from pots with inoculated plants adjacent to control pots. In some cases, the contamination of the controls was associated with wounds in the base of the stem that could have served as a point of entry for the fungus.

The use of a green fluorescent-strain facilitated the differentiation of the inoculated fungus from other Fusarium spp. and other contaminants in the tissue.

In the present work, the inoculated strain was observed in the pedicel of mature seeds as reported in previous research $(2,11,37)$. Pedicel infection may be the primary source of seed transmission and subsequent systemic infection by $F$. verticillioides. Under field conditions, both systemic infection and local infection through silks or insect wounds can lead to infection of pedicels.

Our results confirm that high temperatures favor the development of systemic infection by $F$. verticillioides within maize stalks. The movement of the inoculated strain from stalk internodes into the peduncle and kernels occurred at a low frequency for all reproductive-stage temperature treatments. Resource limitations precluded an experimental design that would allow us to investigate all possible combinations of temperature treatments applied at vegetative versus reproductive growth stages. Further research should address effects of extreme temperatures, interactions between temperature and plant water relations, and further clarify the impacts of temperature at different growth stages on kernel infection by systemic $F$. verticillioides strains.

\section{ACKNOWLEDGMENTS}

This work was supported by Hatch Act and State of Iowa funds through the Iowa Agriculture and Home Economics Experiment Station, Ames, IA We thank Charlotte Bronson, Jennifer Perry, and Amy Wilke, Iowa State University, for their work in preparing the GFP-transformant; Dave Volkers, Iowa State University, for his assistance in maintaining the plants and growth chambers during the experiments; and Palle Pedersen, Iowa State University, Reid Landes, Iowa State University Statistical Consulting Lab, and Adriana Tomas, DuPont Crop Genetics, for their helpful consultations.

\section{LITERATURE CITED}

1. Arino, A. A., and Bullerman, L. B. 1994 Fungal colonization of corn grown in Nebraska in relation to year, genotype and growing conditions. J. Food Prot. 12:1084-1087.

2. Bacon, C. W., and Hinton, D. M. 1996. Symptomless endophytic colonization of maize by Fusarium moniliforme. Can. J. Bot. 74:11951202.

3. Bollero, G. A., Bullock, D. G., and Hollinger, S. E. 1996. Soil temperature and planting date effects on corn yield, leaf area, and plant development. Agron. J. 88:385-390.

4. Bush, B. J., Carson, M. L., Cubeta, M. A., Hagler, W. M., and Payne, G. A. 2004. Infection and fumonisin production by Fusarium verticillioides in developing maize kernels. Phytopathology 94:88-93.

5. Cotten, T. K. 1996. Survival and seed transmission of Fusarium moniliforme, Fusarium proliferatum, and Fusarium subglutinans in maize. MS thesis. Iowa State University, Ames, IA.

6. Desjardins, A. E., and Proctor, R. H. 2001 Biochemistry and genetics of Fusarium toxins. Pages 122-137 in: Fusarium: Paul E. Nelson Memorial Symposium. B. Summerell, J. F Leslie, D. Backhouse, W. L. Bryden, and L. W. Burgess, eds. American Phytopathological Society, St Paul. MN.

7. Doohan, F. M., Brennan, J., and Cooke, B. M. 2003. Influence of climatic factors on Fusarium species pathogenic to cereals. Eur. J. Plant Pathol. 109:755-768.

8. Elford, C. R., and. Shaw, R. H. 1960. The climate of Iowa: Soil temperatures at Ames. Spec. Rep. Agricultural and Home Economics Experiment Station, Iowa State University, Ames, IA.

9. El-Meleigi, M. A., Clafin, L. E., and Raney, R J. 1983. Effect of seedborne Fusarium moniliforme and irrigation scheduling on colonization of root and stalk tissue, stalk rot incidence, and grain yields. Crop Sci. 23:10251028.

10. Etcheverry, M., Torres, A., Ramirez, M. L., Chulze, S., and Magan, N. 2002. In vitro control of growth and fumonisin production by Fusarium verticillioides and $F$. proliferatum using antioxidants under different water availability and temperature regimes. J. Appl. Microbiol. 92:624-632.

11. Foley, D. C. 1962. Systemic infection of corn by Fusarium moniliforme. Phytopathology 52:870-873.

12. Gelderblom, W. C. A., Rheeder, J. P., Leggot, N., Stockenstrom, S., Humphreys, J., Shephard, 
G. S., and Marasas, W. F. O. 2004. Fumonisin contamination of a corn sample associated with the induction of hepatocarcinogenesis in ratsrole of dietary deficiencies. Food Chem. Toxicol. 42:471-479.

13. Kedera, C. J., Leslie, J. F., and Clafin, L. E. 1992. Systemic infection of corn by Fusarium moniliforme. (Abstr.) Phytopathology 82:1138.

14. Kingsland, G., and Wernham, C. 1962. Etiology of stalk rots of corn in Pennsylvania. Phytopathology 52:519-523.

15. Lawrence, E. B., Nelson, P. E., and Ayers, J. E. 1981. Histopathology of sweet corn seed and plants infected with Fusarium moniliforme and F. oxysporum. Phytopathology 71:379-386.

16. Leach, J., Lang, B. R., and Yoder, O. C. 1982. Methods for selection of mutants and in vitro culture of Cochliobolus heterostrophus. J. Gen. Microbiol. 128:1719-1729.

17. Logrieco, A., Mule, G., Moretti, A., and Bottalico, A. 2002. Toxigenic Fusarium species and mycotoxins associated with maize ear rot in Europe. Eur. J. Plant Pathol. 108:597-609.

18. Marasas, W. F. O. 2001. Discovery and occurrence of the fumonisins: A historical perspective. Environ. Health Perspect. 109 (Suppl. 2):239-243.

19. Maric, A. 1981. Fusarium diseases of wheat and corn in Eastern Europe and Soviet Union. Pages 77-93 in: Fusarium: Diseases, Biology and Taxonomy. P. E. Nelson, T. A. Toussoun, and R. J. Cook, eds. Pennsylvania State University Press, State College, PA.

20. Marin, S., Sanchis, V., and Magan, N. 1995. Water activity, temperature and $\mathrm{pH}$ effects on growth of Fusarium moniliforme and Fusarium proliferatum isolated from maize. Can. J. Microbiol. 41:1063-1070.

21. Marin, S., Sanchis, V., Ramos, A. J., Vinas, I., and Magan, N. 1998. Environmental factors, in vitro interactions, and niche overlap between Fusarium moniliforme, $F$. proliferatum, and $F$. graminearum, Aspergillus and Penicillium species from maize. Mycol. Res. 102:831-837.
22. McGee, D. C. 1988. Maize Diseases: A Reference Source for Seed Technologists. American Phytopathological Society, St. Paul, MN.

23. Miller, D. J. 2001. Factors that affect the occurrence of fumonisins. Environ. Health Perspect. 109(Suppl. 2):321-324.

24. Miller, J. D., Savard, M. E., Schaafsma, A. W., Seifert, K. A., and Reid, L. M. 1995. Mycotoxin production by Fusarium moniliforme and Fusarium proliferatum from Ontario and occurrence of fumonisin in the 1993 corn crop. Can. J. Plant Pathol. 17:233-239.

25. Munkvold, G. P. 2003. Mycotoxins in cornoccurrence, impact and management. Pages 1-71 in: Corn: Chemistry and Technology. 2nd ed. P. White and L. Johnson, eds. American Association of Cereal Chemists, St. Paul, $\mathrm{MN}$.

26. Munkvold, G. P., and Carlton, W. M. 1997. Influence of inoculation method on systemic Fusarium moniliforme infection of maize plants grown from infected seeds. Plant Dis. 81:211-216.

27. Munkvold, G. P., McGee, D. C., and Carlton, W. M. 1997. Importance of different pathways for maize kernel infection by Fusarium moniliforme. Phytopathology 87:209-217.

28. Nash, S. M., and Snyder, W. C. 1962. Quantitative estimations by plate counts of propagules of the bean root rot Fusarium in field soils. Phytopathology 52:567-572.

29. Nelson, P. E. 1992. Taxonomy and biology of Fusarium moniliforme. Mycopathologia 117:29-36.

30. Ooka, J. J., and Kommedahl, T. 1977. Wind and rain dispersal of Fusarium moniliforme in corn fields. Phytopathology 67:1023-1026.

31. Pascale, M., Visconti, A., and Chelkowsky, J. 2002. Ear rot susceptibility and mycotoxin contamination of maize hybrids inoculated with Fusarium species under field conditions. Eur. J. Plant Pathol. 108:645-651.

32. Reid, L. M., Nicol, R. W., Ouellet, T., Savard, M., Miller, J. D., Young, J. C., Stewart, D. W., and Schaafsma, A. W. 1999. Interaction of Fusarium graminearum and F. moniliforme in maize ears: Disease progress, fungal biomass, and mycotoxin accumulation. Phytopathology 89:1028-1037.

33. Ritchie, S. W., Hanway, J. J., and Benson, G. O. 1992. How a corn plant develops. Iowa State Univ. Ext. Spec. Rep. 48.

34. Shelby, R. A., White, D. G., and Bauske, E. M 1994. Differential fumonisin production in maize hybrids. Plant Dis. 78:582-584.

35. Srobarova, A. Moretti, A. Ferracane, R Ritieni, A., and Logriego, A. 2002. Toxigenic Fusarium species of Liseola section in preharvest maize ear rot, and associated mycotoxins in Slovakia. Eur. J. Plant Pathol. 108:299 306.

36. Stewart, D. W., Reid, L. M., Nicol, R. W., and Schaafsma, A. W. 2002. A mathematical simulation of growth of Fusarium in maize ears after artificial inoculation. Phytopathology 92:534-541.

37. Sumner, D. R. 1968. Ecology of corn stalk rot in Nebraska. Phytopathology 58:755-760.

38. Vigier, B., Reid, L. M., Seifert, K. A., Stewart, D. W., and Hamilton, R. I. 1997. Distribution and prediction of Fusarium species associated with maize ear rot in Ontario. Can. J. Plant Pathol. 19:60-65.

39. Voss, K. A., Howard, P. C., Riley, R. T., Sharma, R. P., Bucci, T. J., and Lorentzen, R J. 2002. Carcinogenicity and mechanism of action of fumonisin $\mathrm{B}_{1}$ : A mycotoxin produced by Fusarium moniliforme ( $=F$. verticillioides) Cancer Detect. Prev. 26:1-9.

40. Wilke, A. L., Bronson, C. R., Tomas, A., and Munkvold, G. P. 2007. Seed transmission of Fusarium verticillioides in maize plants grown under three different temperature regimes. Plant Dis. 91:1109-1115.

41. Yates, I. E., Arnold, J. W., Hinton, D. M., Basinger, W., and Walcott, R. R. 2003. Fusarium verticillioides induction of maize seed rot and its control. Can. J. Bot. 81:422-428. 